\title{
¿Qué, cuánto y dónde se escribe en lingüística?: Análisis de la producción científica de los becarios de doctorado CONICYT-Chile*
}

\author{
Ángel Valenzuela** \\ David Pino-Alonso***
}

\begin{abstract}
Resumen
La producción científica a través de la publicación de artículos se ha convertido en un criterio de validación y cualificación investigativa en la formación de capital humano avanzado. Por esto, el objetivo del presente estudio fue analizar la producción científica en el área de la lingüística, particularmente la generada por los egresados de doctorado financiados por el programa de becas de Chile. En cuanto a la metodología, se contempló como unidad de análisis las publicaciones realizadas por los becarios cuyo egreso estuvo entre los años 2008 y 2015. Los resultados indican que las dinámicas de la producción científica son relativamente estables, produciéndose alzas al finalizar el proceso de formación doctoral.
\end{abstract}

Palabras clave: Producción científica, lingüística, Formación de capital humano avanzado.

\section{What, how many and where is it Written in Linguistics? Analysis of the Scientific Production of Doctorate Scholarchips Conicyt-Chile}

\begin{abstract}
Scientific production through the publication of articles has become a criterion of validation and research qualification in the formation of advanced human capital. Therefore, the objective of this study was to analyze the scientific production in linguistics, particularly that generated by doctoral graduates financed by the scholarship program of Chile. Regarding the methodology, the publications carried out by the fellows whose discharge was between 2008 and 2015 were considered as the unit of analysis. The results indicate that the dynamics of scientific production are relatively stable, with rises occurring at the end of the training process doctoral.
\end{abstract}

\footnotetext{
* $\quad$ Este artículo se inscribe en el Proyecto de Investigación doctoral "Monitoreo y control metacognitivo en situaciones de lectura y escritura en contextos académicos". Se agradece la colaboración de Felipe Bustamante G. y Carol Becerra S., estudiantes de Ped. en lengua castellana y comunicación, Univ. Autónoma de Chile.

** Chileno. Doctor (c) en Ciencias Humanas. Universidad de Talca, Chile. Becario CONICYT-PFCHA/ Doctorado Nacional/2017-21171977. anvalenzuela@utalca.cl

*** Chileno. Máster en Iniciación a la Investigación en Textos de la Antigüedad Clásica y su pervivencia. Universidad de Salamanca. Docente de la Universidad Autónoma de Chile, Talca, Chile. dapino@ uautonoma.cl
} 
Keywords: Scientific production, Linguistics, Formation of advanced human capital.

\section{Presentación}

En la formación de capital humano avanzado, la producción científica a través de la publicación de artículos científicos o papers se ha convertido en un criterio de validación y cualificación investigativa, independiente de la disciplina o área de investigación (Carlino, 2006, 2012; Lillis, 2003; Rawat \& Meena, 2014). Incluso, en los últimos años goza de una aceptación mayor que el género per se de las instancias doctorales, como ha sido la elaboración del género Tesis (Rawat \& Meena, 2014). Esto sucede, principalmente, porque a través de la publicación de un artículo de investigación se posibilita una mayor visibilidad de los hallazgos, además de entregar prestigio entre la comunidad investigativa (Valderrama, 2012), pero sobre todo, se transforma en un mecanismo de suficiencia, clave en la obtención de financiamiento si el objetivo es realizar -o continuar- un proyecto investigación de mayor envergadura. ${ }^{1}$

No obstante, surgen cuestionamientos sobre las prácticas que se adoptan en la divulgación del conocimiento científico: Publish or perish, predatory journal, etc. (Cordeiro \& Lima, 2017; Goodman, 2018)Butler 2013. Pese a ello, la publicación de un paper en una revista de corriente principal, continúa siendo la principal instancia de producción y difusión, y un espacio fundamental en la formación de capital humano avanzado (Cordeiro \& Lima, 2017; Fraser, 2017; Lotka, 1926; Takrouri, 2016; Walsh, 2011). Esto se debe a que la publicación en un journal goza de confiabilidad como canal de comunicación de la investigación, por cuanto las mismas, suponen una evaluación rigurosa y exhaustiva por parte de los miembros de la comunidad científica (Salas et al., 2017; Valderrama, 2012).

En el área de lingüística, no hay mayores diferencias en cuanto a las dinámicas de la difusión científica. En ese sentido, desde que en el segundo decenio del siglo XIX los estudios sobre el lenguaje adaptaran un carácter eminentemente científico (Campbell, 2008; Cerny, 1998;

Por ejemplo, en Chile este es uno de los principales criterios de adjudicación de los Fondos de Investigación Científica de Conicyt. 
Mahecha, 2008), estos han utilizado las formas de transmisión convencionales de la ciencia moderna. Sin embargo, no es hasta con la publicación del libro Language de Sapir en 1921 y del Cours de linguistique générale de Saussure en 1915, que el campo de la lingüística comenzó a tomar forma como disciplina independiente. Así, cuando la Linguistic Society of America (LSA) publicó en el año 1925 la revista científica Language, marcó un hito en la investigación en el área, dando un carácter científico a los estudios sobre el lenguaje humano.

Actualmente, la lingüística, al igual que un sinnúmero de otras disciplinas, amplía sus fronteras a nuevos temas, enfoques y metodologías, haciendo de ella un lugar de encuentro a psicólogos cognitivos, psicólogos sociales, sociólogos, neurólogos, especialistas en didáctica, educadores, entre otros; lo que guarda relación en que existan alrededor de 720 revistas en la categoría Language and Linguistics de Scimago Journal (Scopus, 2018), con más de 17.000 documentos académicos, las cuales no solo permiten la visibilización de la investigación científica en el área de la lingüística, sino también dan un carácter propio a la disciplina a partir de un posicionamiento interdisciplinario.

Por lo anterior, en la presente investigación se intentó identificar las dinámicas investigativas que se priorizan en determinados momentos en el mundo científico: métodos, autores, intereses, etc. En ese afán, el presente estudio, analiza la producción científica en el área de la lingüística, particularmente la generada por los egresados de doctorado financiados por el programa de becas de la Comisión Nacional de Investigación Científica y Tecnológica de Chile (Conicyt). De este modo, se espera responder a: ¿qué temáticas se han investigado en los últimos años en el campo de la lingüística?, ¿cuánto es la producción científica en el campo de la lingüística?, y ¿en qué revistas científicas se han publicado las investigaciones en el área de la lingüística?

\section{La producción científica en las revistas de investigación}

A partir de los avances de científicos y tecnológicos, se generan nuevos conocimientos y nuevas formas de enfrentarse al mundo, apareciendo también nuevos desafíos (OECD, 2014; Tasioulas \& Vayena, 2016). En ese sentido, uno de los principales retos que ha enfrentado la ciencia y la tecnología ha sido la divulgación de los hallazgos de la investigación científica (OECD, 2014). Por ello, a partir del posicionamiento de los paradigmas 
de la ciencia moderna, surgieron repositorios académicos que permiten dar a conocer a la comunidad científica y en general, los descubrimientos de una investigación, permitiendo, además, una sistematización de los procesos investigativos (Sánchez-Tarragó et al., 2016).

De este modo, estos repositorios produjeron lo que conocemos como revistas de divulgación científica o journal. En cuanto a estas, su origen se remonta al siglo XVII, cuando bajo el alero de los grupos de investigación de la Académie Royale des Science de Francia y la Royal Society de Gran Bretaña, se conformó un método de expresión crítica a través de comentarios, evaluaciones y juicios críticos sobre los métodos y resultados de las investigaciones que se realizan, mediante la validación o rechazo de la pertinencia de los hallazgos (Mendoza \& Paravic, 2006) ${ }^{2}$. No obstante, no es hasta 1752, con la revista Philosophical Transactions, que los criterios de revisión de un comité evaluativo, como los que existen hoy en día, determinarían si un artículo era lo suficientemente adecuado para ser admitido y publicado por un journal, añadiendo el sesgo de aceptación o rechazo por parte de la comunidad científica (Mendoza \& Paravic, 2006), confiriendo, de este modo, una mayor confianza al discurso científico.

A partir de ello, la difusión del conocimiento científico, se comenzó a posicionar como un discurso confiable, cercano a la "verdad", muy distinto a la retórica de la cotidianeidad, de la política partidista o de la publicidad (Gooch, Vasalou, \& Benton, 2017; Hyland, 2011; Lillis, 2003). Sin embargo, tal vez por una mercantilización del quehacer científico, este discurso sufre de cuestionamientos que socavan, en cierto sentido, el mismo carácter científico: prácticas que se adoptan en la divulgación del conocimiento científico a partir de las acciones realizadas por algunas editoriales, journals, entidades académicas, e investigadores ${ }^{3}$. Pese a ello, igualmente la producción científica a través de artículos publicados en revistas científicas sigue suponiendo una evaluación rigurosa y exhaustiva de los contenidos, métodos y hallazgos de una investigación (Borrego \& Urbano, 2006; Zuin, Bianchetti, Zuin, \& Bianchetti, 2015)prestando un especial interés a los títulos de Ciencias Sociales y Humanidades. En primer lugar, se examinan las dificultades de aplicación, en estas disciplinas, de métodos bibliométricos de repercusión basados en el

Entre estas primeras revistas se encuentran el Journal de Sçavans, la Miscellanea Curiosa, el Journal des Nouvelles Découvertes sur Toutes les Parties de la Medicine, y el Philosophical Transactions of the Royal Society.

3 Publish or perish, predatory journal, etc. (Bolshete, 2018; Goodman, 2018). 
análisis de citas con datos obtenidos de las bases de datos de Thomson Scientific (ex ISI.

Por esto, en la formación de capital humano avanzado, independiente del campo disciplinar, la producción científica a través de la publicación de artículos científicos se ha vuelto una instancia de certificación de competencias investigativas. De este modo, se ha generado, en los últimos años, un vuelco en cuanto a la atención de las formas de producción científica, (Pacheco, 2015; Parodi, 2012)the research objectives are to identify and to describe the academic discourse genres based on a corpus of 3,147 texts collected in six disciplines in Social Sciences and Humanities and Basic Sciences (PUCv-2010 Corpus; con el fin de desdibujar lo local en lo global por medio de la universalización y democratización del saber (Salas et al., 2017). Esto llevó a que las revistas científicas experimentaran procesos de transformación tanto en la visibilidad de sus contenidos (apropiación académica) como en la difusión (apropiación social) de los mismos (Salas et al., 2017), alcanzando también a las orgánicas disciplinares, las cuales han tenido que consolidar espacios y temáticas que permitan acrecentar el conocimiento científico, lo que hace de esta una acción perentoria en las instancias de formación doctoral.

A partir de lo anterior, hay un reposicionamiento del quehacer científico, marcado en ocasiones por los vaivenes propios de la Modernidad. Esto ha llevado a que, cada vez más, surja una enorme cantidad de información desde la producción científica (Bolshete, 2018; Campanario, 2018), lo cual, en variadas ocasiones, limita el propio accionar investigativo (Sarewitz, 2016). A pesar de esto, como se señaló anteriormente, la producción científica, a través de la publicación de artículos de investigación en revistas científicas, sigue siendo una instancia de suficiencia investigativa, clave en el proceso de formación de capital humano avanzado.

\section{El espacio disciplinar de la lingüística y la productividad científica}

Una disciplina es una forma de organización social que genera nuevas ideas y hallazgos a partir de preguntas de investigación, certificando y promoviendo ese conocimiento generado a una comunidad en particular y en general (Jacobs, 2013; Navarro et al., 2016)from major universities to the National Institutes of Health. In Defense of Disciplines presents a 
fresh and daring analysis of the argument surrounding interdisciplinarity. Challenging the belief that blurring the boundaries between traditional academic fields promotes more integrated research and effective teaching, Jerry Jacobs contends that the promise of interdisciplinarity is illusory and that critiques of established disciplines are often overstated and misplaced $\backslash$ "--- Part 1. Academic disciplines, specialization, and scholarly communication -- The critique of disciplinary silos -- Dynamic disciplines -- Specialization, synthesis, and the proliferation of journals / (coauthored with Rebecca Henderson. De este modo, la producción científica a partir de artículos de investigación forma parte central de las otras dinámicas de los campos científicos como las instancias de formación académica y profesional, los congresos, seminarios, entre otros; en donde no solo se promueve la integración disciplinar, sino también la legitimación, el control y la reproducción de los hallazgos, métodos y teorías (Navarro et al., 2016)aún son escasas las investigaciones sistemáticas sobre la configuración de este espacio disciplinar. Con el propósito de llenar este vacío, el presente artículo identifica continuidades y contrastes locales e históricos a partir de un conjunto de variables (autores, idiomas, focos, temas, orientaciones, niveles, áreas, géneros y tipos.

De este modo, a pesar de la aparición algo tardía en la historia del saber de la lingüística como ciencia con un objeto de estudio claro (Saussure, 2005), la reflexión y discusión sobre los hechos del lenguaje ha sido una preocupación de filósofos y pensadores que han visto en el lenguaje una capacidad única de reflejar el pensamiento y permitir la comunicación, de modo que tratados referidos a la lenguas concretas, como el sánscrito o las lenguas sumerias, ya se encuentran en textos que sobrepasan el milenio de antigüedad (Robins, 1997).

Durante siglos, los estudios relativos a las lenguas y su conformación estructural fue una tarea propia de la "Gramática" entendida en un sentido muy amplio, la cual abarcaba la morfología, la sintaxis, la ortografía, la prosodia y rudimentos de fonética y fonología de un modo indirecto a través del análisis de los textos tal como se aprecia en las principales gramáticas de la tradición clásica, como las de Dionisio Tracio, Prisciano y Donato (Galino, 1960); y que reflejan una tradición en la que lo importante estaba en las "autoridades" del lenguaje, tales como poetas y prosistas en general.

Con el paso del tiempo, las gramáticas se hicieron parte fundamental del currículum escolar europeo y se convirtieron en importantes 
manuales que servían para utilizar la lengua con el fin de conseguir efectos persuasivos o literarios que en poco se relacionaban con las manifestaciones culturales de la lengua en cuanto a una interacción social permanente. Así, la "gramática" en un sentido amplio -sobre todo en el periodo alejandriano- fue una disciplina auxiliar de la crítica textual e interpretación de textos escritos (Pfeiffer, 1981). Asimismo, la tradición científica ha estado de acuerdo en determinar un punto de inflexión en las propuestas del Cours de linguistique générale, una obra que contiene apuntes de una propuesta que posiciona a la lingüística dentro de una visión positivista, con un objeto de estudio aislable por parte del investigador: la langue (Saussure, 2005); la cual se inscribe dentro un sistema que permite establecer estándares que sean válidos para todos los códigos que el ser humano ha creado para conseguir la comunicación.

La obra de Saussure, a pesar de ser superada en muchos aspectos, sigue siendo un referente principal de las ciencias del lenguaje y algunas de sus dicotomías conservan pleno valor (Molina, 2017). Otra consecuencia importante de la definición de la lingüística científica fue la especialización cada vez mayor de otras disciplinas que estudian el lenguaje humano, como la fonética, la fonología, la semántica, la pragmática, entre otras, las cuales se independizaron de la gramática y formaron sus propias corrientes teóricas y metodologías, que hasta ahora se sitúan como disciplinas independientes unas de otras (Escandell, 1993; Riemer, 2010; Zsiga, 2013).

Sin embargo, una permanente especialización trae consigo nuevamente la necesidad de poner en relación diferentes disciplinas que, buscando su lugar en la ciencia, no son capaces por sí mismas de abarcar todos los aspectos del lenguaje humano o de una lengua en particular. En este sentido, actualmente estas disciplinas se entienden como transdisciplinarias, concepto complejo que da cuenta de un movimiento dialéctico entre diversas áreas del saber que permiten conformar una aprehensión mayor de la realidad (Martínez-Miguélez, 2007). En esta etapa, la lingüística se inscribe como un puente entre las ciencias sociales, humanas e incluso biológicas, si consideramos los aspectos fisiológicos e incluso cognitivos que son parte de la adquisición y la producción de la lengua. Por ello, gracias a este permanente diálogo con disciplinas afines -y a veces no tan afines-, como la fonoaudiología, la psicología, la neurología, la fisiología y hasta el derecho, por nombrar algunas, surgen áreas de conexión como la sociolingüística, psicolingüística, 
neurolingüística, la lingüística aplicada (Ahlsén, 2006; Garman, 1990; Moreno Fernández, 1998; Schmitt, 2002), como algunos de los ejemplos más clásicos. Esto permite posicionar a la lingüística como una disciplina moldeable a este tipo de trabajo transdisciplinar.

Por lo anterior, es que la productividad científica en el área de la lingüística trasciende las convenciones clásicas de posicionar un objeto de estudio en una disciplina. Tal vez por esto, en la categoría Language and Linguistics de Scimago Journal (Scopus, 2018), haya 720 revistas científicas, estando entre las primeras, de acuerdo al índice $\mathrm{H}$, revistas que no necesariamente se adscriben a la lingüística propiamente tal, como por ejemplo Cognitive Psychology, Cognition, Journal of Cognitive Neuroscience, Artificial Intelligence, Journal of Experimental Psychology: Learning Memory and Cognition, entre otras. De este modo, se manifiesta el carácter interdisciplinario y transdisciplinar que adquiere el lenguaje humano.

En cuanto a las dinámicas de investigación en Latinoamérica en el área de la lingüística, hay semejanzas con las dinámicas de otras regiones del mundo, las cuales se pueden constatar no solo en la publicación de artículos, sino también en espacios como seminarios, congresos, conferencias, entre otros. Sin embargo, aún la productividad científica en la región en lo cuantitativo es baja. En el año 2016 se publicaron 565 artículos en 24 revistas de la base de datos de Scimago Journal, lo cual corresponde al 3,2\% del total de publicaciones que se realizaron considerando la categoría Language and Linguistics.

\section{Método}

El presente estudio contempló como unidad de análisis 73 artículos publicados en 39 revistas científicas entre los años 2003 y 2017, correspondientes a 25 egresados de doctorados financiados por el programa de Becas de Conicyt. No obstante, inicialmente se consideraron, desde la base de datos en la página web de Conicyt ${ }^{4}, 33$ egresados de doctorado (20 mujeres y 13 hombres), los cuales cumplían con los criterios de inclusión: realizar estudios doctorales en el área de la lingüística, cuyo egreso fuera entre el año 2008 y el 2015. Sin embargo, no se evidencia productividad científica a través de artículos de investigación en 8 de ellos (24,2\%).

www.conicyt.cl 
En cuanto a la recolección de los documentos, esta fue realizada entre los meses de julio y agosto del año 2017, utilizando un buscador general (Google), una comunidad virtual académica (Researchgate) y bases de datos científicas (Scielo, Scopus y WoS). Para la búsqueda, primeramente, se consideraba el nombre completo del investigador y luego se realizaban distintas combinaciones con el nombre, pasando desde el buscador general hasta las bases de datos científicas, tal cual se consigna en la imagen 1. Además, cabe señalar que solo se consideró la productividad científica correspondiente a artículos de investigación en una revista científica, por lo tanto, se excluyeron artículos de reseña, editoriales, conferencias, homenajes, entrevistas, libros, capítulos de libros y actas de congreso.

Imagen 1. Recolección y construcción de la base de datos.

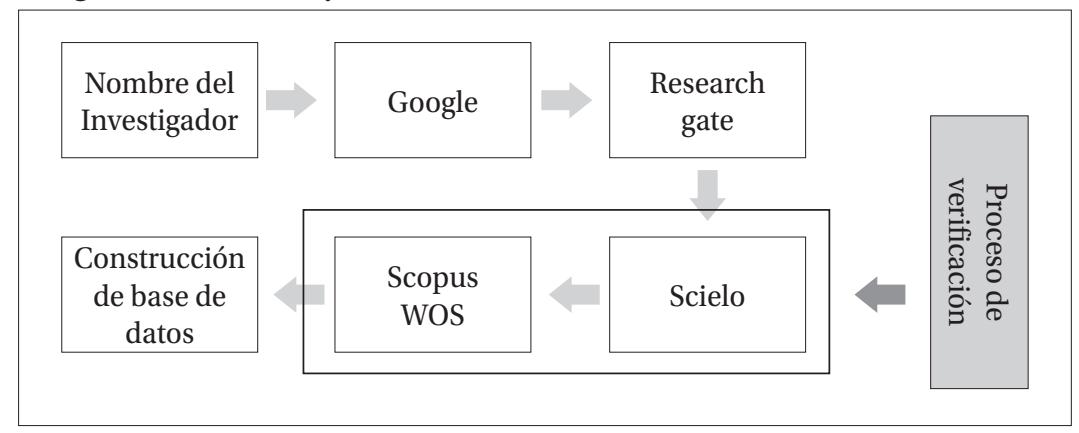

En cuanto al análisis estadístico, este es de carácter eminentemente descriptivo y se realiza a partir tanto de la información de los becarios como del análisis de los artículos publicados, a través de una descripción sistemática de un conjunto de indicadores generales: nombre de la revista o journal, año de publicación, idioma, colaboración, temáticas y metodologías de investigación utilizadas (ver Tabla 1). Estos indicadores seleccionados corresponden a los utilizados frecuentemente en los estudios bibliométricos en distintas disciplinas (Glänzel \& Schoepflin, 1999; Salas et al., 2017)considerando algunos elementos históricos que contextualizan su trayectoria, para describir sus principales características y evaluar su evolución cronológica. Para ello se construyó una base de datos con la totalidad de publicaciones de la revista (25 volúmenes y 48 números, en los que se describe la frecuencia de artículos en función de un conjunto de variables criterios y sus respectivos niveles o categorías (por ejemplo: 1, 2, 3 autores; primer autor o no; metodología cuantitativa, cualitativa, mixta). 
En el análisis de las áreas temáticas de los artículos, se consideró la clasificación utilizada en los congresos de la Sociedad Chilena de Lingüística (Sochil) en las dos últimas versiones (2015 y 2017). La asignación de un artículo a un área en particular se realizó a través del juicio de dos pares evaluadores (docentes universitarios en el área de lingüística y temas afines), siguiendo el procedimiento de doble ciego. En los casos que existió desacuerdo en la clasificación temática, se debatió en una segunda etapa y se acordó elegir la categoría más representativa, siendo este procedimiento utilizado también para la asignación de las metodologías.

En la descripción de los indicadores de impacto de la revista, se utilizó un ranking elaborado por Scimago Research Group (2007) que clasifica a las revistas en cuartiles (Q1, Q2, Q3, Q4) de acuerdo con la cantidad de citas y de artículos publicados en la revista, siendo los primeros cuartiles los que tienen mayor impacto. Para las revistas que no estaban indexadas en SJR, se consideró la indexación a otras bases de datos, como Scielo, Dialnet y Latindex. En este punto cabe señalar que se la asignación a un cuartil o base de datos en específico se realizó considerando el año de la publicación del artículo y no el ranking actual de la revista.

Tabla 1. Descripción de los indicadores.

\begin{tabular}{|l|l|}
\hline Indicadores & Descripción \\
\hline $\begin{array}{l}\text { Número de artículos } \\
\text { publicados }\end{array}$ & $\begin{array}{l}\text { Número de artículos publicados entre los becarios doc- } \\
\text { torales de Conicyt. }\end{array}$ \\
\hline Revista o journal & $\begin{array}{l}\text { Nombre de la revista en donde se publicó el artículo de } \\
\text { investigación. }\end{array}$ \\
\hline $\begin{array}{l}\text { Ranking de revista } \\
\text { (Indexación) }\end{array}$ & $\begin{array}{l}\text { Corresponde al indicador de impacto de la revista, a partir } \\
\text { de un ranking elaborado por SCImago Research Group } \\
\text { (2007). También se consideran las revistas que tienen otro } \\
\text { tipo de indexación como Scielo, Dialnet y Latindex. }\end{array}$ \\
\hline Año de publicación & Corresponde al año de la publicación del artículo. \\
\hline Idioma & Corresponde al idioma de publicación del artículo. \\
\hline Número de autores & Número de autores firmantes por el artículo. \\
\hline Temáticas & $\begin{array}{l}\text { Corresponde a las áreas temáticas de investigación de } \\
\text { los artículos de acuerdo con la clasificación de los jueces. } \\
\text { Se consideraron las áreas propuesta por la Sociedad } \\
\text { Chilena de Lingüística (Sochil). }\end{array}$ \\
\hline Metodologías & $\begin{array}{l}\text { Tipo de metodología de investigación empleada en los } \\
\text { artículos, de acuerdo a la clasificación por jueces. }\end{array}$ \\
\hline
\end{tabular}




\section{Resultados}

Los resultados dan cuenta sobre la productividad científica de los becarios de doctorado Conicyt (cantidad de publicaciones, revistas, etc.) y de los indicadores asociados a los artículos publicados en las revistas científicas (colaboración, temáticas, metodologías, idioma, indicadores de impacto). De esta forma, se intenta responder a las preguntas iniciales ¿qué, cuánto y dónde se escribe en lingüística?

\subsection{Productibilidad}

Como se señaló anteriormente, se encontraron 73 artículos publicados en revistas indexadas, correspondiente a 25 investigadores. Sin embargo, dos de los artículos tienen como coautoría a egresados de doctorado en área de lingüística del periodo estudiado. Por esto, se consideró contabilizar de manera doble dos artículos para dar una correspondencia entre un investigador y un escrito. Así, el corpus analizado consistió en 75 artículos, dando como promedio 3 artículos publicados por investigador. Sin embargo, al considerar a todos los becarios el promedio descendió a 2,2.

En cuanto a la variable primer autor, la presencia de los becarios Conicyt es de un $69,3 \%$. En ese sentido, cabe señalar que casi la mitad de las publicaciones que conforman el corpus tiene como firmante solo a un autor $(45,3 \%)$, de los cuales un $24 \%$ son hombres y un $21,3 \%$ son mujeres. A pesar de ello, se observa una mayor presencia de mujeres en el total de publicaciones: 43 artículos (57,3\%) tienen como autores firmantes a 16 mujeres y 32 artículos (42,7\%) a 9 hombres. No obstante, hay una mayor proporción de escritos publicados por hombres: 3,55 frente a 2,68.

Con relación a la indexación, un $74,7 \%$ de los escritos está publicado en una revista con indexación Scopus o WoS, mientras que 14,7\% está publicado en revistas con indexación SciELO. En cuanto al idioma, el $80 \%$ de las publicaciones están en español y solo un $20 \%$ en inglés. Sobre este último, solo hay 1 artículo adscrito a un investigador que egresó de un doctorado nacional y que está publicado en inglés. Así, del $80 \%$ de las publicaciones en español, un $66,7 \%$ corresponde a egresados de programas doctorales en Chile. 
Tabla 2. Descriptivos de artículos seleccionados.

\begin{tabular}{|l|c|c|c|}
\hline & Total & Hombres & Mujeres \\
\hline Artículos & & & \\
\hline - N & 75 & $32(42,7 \%)$ & $43(57,3 \%)$ \\
\hline - Autores & 25 & $9(36 \%)$ & $16(64 \%)$ \\
\hline Indexación & & & \\
\hline - Scopus / WoS & $56(74,7 \%)$ & $19(25,4 \%)$ & $37(49,3 \%)$ \\
\hline - SciELO & $11(14,7 \%)$ & $7(9,3 \%)$ & $4(5,3 \%)$ \\
\hline - Otra & $8(10,7)$ & $6(8 \%)$ & $2(2,7 \%)$ \\
\hline Idioma & & & \\
\hline - español & $60(80 \%)$ & $26(34,7 \%)$ & $34(45,3 \%)$ \\
\hline - inglés & $15(20 \%)$ & $6(8 \%)$ & $9(12 \%)$ \\
\hline Primer autor & $52(69,3 \%)$ & $25(33,3 \%)$ & $27(36 \%)$ \\
\hline
\end{tabular}

Otro de los elementos claves en los indicadores de productividad corresponde al periodo en el cual se realizan las publicaciones de los artículos. De este modo, es posible constatar que, si bien hay una tendencia a publicar posterior al egreso, hay cierta estabilidad en los periodos de publicación. Asimismo, el periodo entre 0 y 2 años después del egreso, tiene los índices mayores de publicación (32\%), no obstante, las publicaciones realizadas antes del egreso también son relevantes $(29,3 \%)$. En cuanto al número de firmantes en los artículos publicados antes del egreso, un $54,5 \%$ corresponde a artículos en colaboración, lo cual se invierte luego del egreso, ya que entre 0 y 2 años después del egreso, un $54,2 \%$ de las publicaciones tienen como firmante solo a un autor. Lo mismo sucede entre los 3 y 4 años después del egreso, con un $55 \%$ de las publicaciones. Esto se invierte después del quinto año o más de egreso, ya que el promedio de autores por publicación es sobre 3. Esto refuerza la idea de que la investigación en lingüística se realiza en grupos reducidos y en variadas ocasiones de manera individual, contraria a la tendencia de otras disciplinas como psicología o las ciencias de la salud, que son más proclives a la colaboración entre investigadores (Salas et al., 2017; Xie, Li, Li, Duan, \& Ouyang, 2018). 


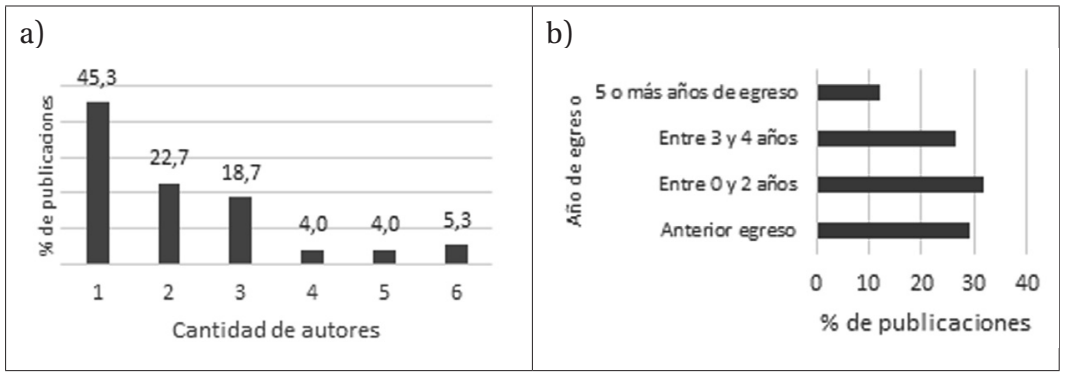

Gráfico 1. Indicadores de productividad. a) porcentaje de publicaciones de los becarios por cantidad de autores firmantes del artículo; b) año de egreso del doctorado por el porcentaje de artículos publicados.

Por último, en relación con los journals en donde se han publicado las investigaciones de los egresados de doctorado, un $64 \%$ corresponde a revistas nacionales, siendo la revista Literatura y Lingüística la que tiene mayor presencia con un $18,7 \%$, siguiéndole la revista Onomazéin y la Revista de Lingüística Aplicada de la Universidad de Concepción con un $10,7 \%$ cada una. Destaca que el $92 \%$ de los egresados haya publicado a lo menos un artículo en revistas con indexación Scopus o WoS durante este periodo, no como una medida necesariamente de calidad, sino como un elemento distintivo, ya que esta es una variable por considerar en la postulación a fondos de investigación de mayor envergadura.

Gráfico 2. Porcentaje de Revistas con contribuciones de becarios Conicyt.

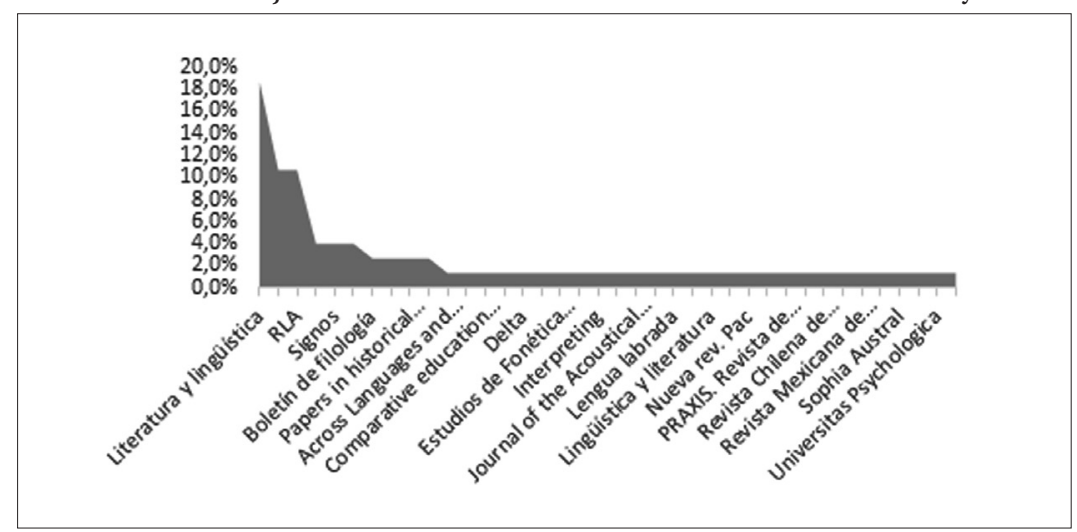

\subsection{Temática y metodologías}

En la clasificación temática se muestra el número de artículos publicados correspondientes a 13 categorías de clasificación utilizadas (Tabla 3). En términos globales, hay una predominancia de publicaciones cuya 
temática son Fonética y fonología con un 22,7\%, Gramática con un 18,7\%, Lingüística aplicada con 14,7\%. En contraparte, se evidencia una menor proporción de estudios dirigidos a áreas como Lingüística cognitiva, Semántica y pragmática, Teorías lingüísticas, y Lexicología y terminología, todas con un $2,7 \%$. En cuanto al tipo de indexación y área temática, destaca que en las revistas clasificadas como Q2, la mayor presencia corresponde a artículos cuyos temas se circunscriben a lingüística aplicada, gramática, y fonética y fonología. Asimismo, destaca que, de los artículos publicados en el área de gramática, ninguno está en revistas clasificadas como Q1. También resalta el área semántica y pragmática, ya que, de los dos artículos publicados, uno tiene presencia en una revista de alta selectividad.

En el caso de la clasificación metodológica, se presenta la frecuencia de artículos en cuatro categorías: cuantitativa, cualitativa, mixta y estudios teóricos y otros. Aquí, hay una alta presencia de estudios con un enfoque cuantitativo (33,3\%), cuyos temas se concentran principalmente en lingüística aplicada, gramática, y fonética y fonología con 5 artículos cada uno. La metodología cualitativa también tiene una alta presencia con un 25,3\%, siendo fonética y fonología el tema más recurrente con 8 artículos. Los estudios de carácter teórico también tienen una presencia relevante con un $28 \%$, siendo los artículos vinculados a la investigación en gramática los más representativos. Por último, los estudios con métodos mixtos tienen una presencia menor con un $13,3 \%$, vinculados principalmente a la sociolingüística y dialectología.

Al considerar el tipo de indexación y metodología, destaca que los estudios con un carácter eminentemente teórico tienen una presencia en todos los tipos de indexación, menos en las revistas clasificadas como Q1. Y, por el contrario, las revistas clasificadas en este cuartil solo consideraron artículos con metodologías cuantitativas y cualitativas (3 artículos cada uno). En cuanto a los primeros, el $80 \%$ se concentra en publicaciones con indexación Q2 y Q3.

Asimismo, con el fin de identificar la evolución tanto de las temáticas como de las metodologías, se realizó una distribución temporal en tres períodos, los cuales permiten visualizar, a grandes rasgos, los intereses temáticos de quienes se formaron como investigadores en el área de la lingüística. Por ejemplo, en la Tabla 3 se puede visualizar una inclinación en los últimos años a una línea más práctica (lingüística aplicada y educación). Asimismo, hay una presencia, si bien escasa, 
demostrativa de enfoques temáticos interdisciplinarios como la psicolingüística, neurolingüística o lingüística cognitiva. Sobre los enfoques metodológicos, considerando los periodos de tiempo, no hay una mayor dominancia de uno por sobre otro, sin embargo, se entrevé una disminución de los estudios eminentemente teóricos.

Tabla 3. Temáticas y Metodologías de investigación por período.

\begin{tabular}{|l|c|c|c|c|}
\hline \multicolumn{4}{|c|}{ Período } & \\
\hline Temática & & $2011-2015$ & $\mathbf{2 0 1 6 - 2 0 1 7}$ & Total \\
- Psicolingüística y neurolingüística & 0 & $2(2,7 \%)$ & $1(1,3 \%)$ & $3(4 \%)$ \\
- Lingüística cognitiva & $1(1,3 \%)$ & 0 & $1(1,3 \%)$ & $2(2,7 \%)$ \\
- Sociolingüística y dialectología & 0 & $5(6,7 \%)$ & $2(2,7 \%)$ & $7(9,3 \%)$ \\
- Etnolingüística & $1(1,3 \%)$ & $2(2,7 \%)$ & 0 & $3(4 \%)$ \\
- Ling. Aplicada & $1(1,3 \%)$ & $6(8 \%)$ & $4(5,3)$ & $11(14,7 \%)$ \\
- Gramática & $2(2,7 \%)$ & $9(12 \%)$ & $3(4 \%)$ & $14(18,7 \%)$ \\
- Análisis del discurso y Ling. del texto & $1(1,3 \%)$ & $1(1,3 \%)$ & $2(2,7 \%)$ & $4(5,3 \%)$ \\
- Fonética y fonología & $3(4 \%)$ & $8(10,7 \%)$ & $6(8 \%)$ & 17 \\
- Traducción e interpretación & 0 & $3(4 \%)$ & $1(1,3 \%)$ & $(22,7 \%)$ \\
- Semántica y pragmática & 0 & $1(1,3 \%)$ & $1(1,3 \%)$ & $4(5,3 \%)$ \\
- Teorías lingüísticas & 0 & $2(2,7 \%)$ & 0 & $2(2,7 \%)$ \\
- Educación & $1(1,3 \%)$ & $2(2,7 \%)$ & $1(1,3 \%)$ & $2(2,7 \%)$ \\
- Lexicología y terminología & $1(1,3 \%)$ & 0 & $1(1,3 \%)$ & $4(5,3 \%)$ \\
& & & & $2(2,7 \%)$ \\
\hline Metodología & & & & \\
- Cuantitativa & 11 & 41 & 23 & 75 \\
- Cualitativa & $3(4 \%)$ & $16(21,3 \%)$ & $6(8 \%)$ & 25 \\
- Mixta & $3(4 \%)$ & $9(12 \%)$ & $7(9,3 \%)$ & $(33,3 \%)$ \\
- Estudios teóricos y otros & $1(1,3 \%)$ & $6(8 \%)$ & $3(4 \%)$ & 19 \\
& $4(5,3 \%)$ & $10(13,3 \%)$ & $7(9,3 \%)$ & $(25,3 \%)$ \\
& & & & $10(13,3 \%)$ \\
\hline Total & & & & $21(28 \%)$ \\
\hline
\end{tabular}

\section{Discusión y conclusiones}

Los resultados obtenidos nos presentan una panorámica de la producción científica en el área de la lingüística durante los últimos años. Estos nos muestran, en primer lugar, una preocupación por la producción de conocimiento científico en la formación de capital humano avanzado, generando no solo nuevos conocimientos teóricos y aplicados en el área 
de la lingüística, sino que, además, permite dar cuenta del impacto que tienen este tipo de políticas públicas implementadas adecuadamente.

En relación con las preguntas iniciales, cabe señalar que, si bien el $\mathrm{N}$ tal vez no es representativo a los intereses particulares de quienes investigan en el área de la lingüística en Latinoamérica, sí nos entrega ciertas luces sobre las afinidades temáticas y metodológicas. Por ejemplo, no es menor el interés que se demuestra en áreas como la fonética y la fonología o el creciente interés en temáticas orientadas a la lingüística aplicada, las cuales tienen una presencia relevante en los encuentros académicos y científicos: congresos, seminarios, workshops, entre otros.

En relación con las revistas científicas que han publicado los artículos de los investigadores estudiados, si bien estas corresponden principalmente a journals del país de origen del investigador, hay un porcentaje relevante (36\%) de artículos que se han publicado en revistas de otros países (es decir, exceptuando Chile). No obstante, cabe señalar que tal vez esta presencia significativa de artículos en revistas nacionales se podría deber a que entre las primeras 10 revistas del ranking Scimago JR en la categoría language and linguistics en Latinoamérica, hay cuatro que son de origen chileno, siendo esto último, una medida relevante ya que se relaciona directamente con la obtención de financiamiento tanto para la continuación de una investigación como para obtener un incentivo por la publicación.

Un elemento que destaca entre las publicaciones científicas es el bajo porcentaje de artículos publicados en inglés (20\%). Si bien este es un tema sensible entre quienes investigan el lenguaje y principalmente la lengua española, no es menos cierto que el inglés se ha convertido en una lengua franca en los contextos científicos. Con esto no se quiere decir que sea la lengua de la ciencia o con la que se hace ciencia, sino más bien, una lengua promotora, que permite tener un mayor alcance de la investigación, si es que el objetivo fuese ese.

En cuanto a los desafíos, sería interesante analizar en los futuros estudios las dinámicas de la producción científica en otros ámbitos, como, por ejemplo, en congresos, encuentros investigativos. También sería interesante comparar los intereses investigativos al comparar la producción científica generada en Latinoamérica con otras latitudes. En estudios similares a este, sería atrayente integrar las palabras clave, las referencias utilizadas por los investigadores o las colaboraciones entre los investigadores, entre otras. 
Finalmente, cabe mencionar que la investigación solo ha tenido como propósito indagar en las dinámicas de la producción científica en el área de la lingüística, y que por ello se consideró relevante tomar como corpus las publicaciones científicas de los egresados de doctorado financiados por Conicyt.

\section{Referencias bibliográficas}

Ahlsén, E. (2006). Introduction to neurolinguistics. Amsterdam: John Benjamins.

Bolshete, P. (2018). Analysis of thirteen predatory publishers: a trap for eager-to-publish researchers. Current Medical Research and Opinion, 34(1), 157-162. https://doi.org/10.1080/03007995.2017 .1358160

Borrego, Á., \& Urbano, C. (2006). La evaluación de revistas científicas en Ciencias Sociales y Humanidades. Información, Cultura y Sociedad, 14(14), 11-27. Recuperado de http://www.scielo.org. ar/pdf/ics/n14/n14a02.pdf

Campanario, J. M. (2018). Are leaders really leading? Journals that are first in Web of Science subject categories in the context of their groups. Scientometrics, 115(1), 111-130. https://doi.org/10.1007/ s11192-018-2660-7

Campbell, L. (2008). The History of Linguistics. In M. Aronoff \& J. RessMiller (Eds.), The Handbook ofLinguistics. Oxford. UK: Blackwell Publishers Ltd. https://doi.org/10.1002/9780470756409

Carlino, P. (2006). La escritura en la investigación (Documentos de trabajo No. 19, Vol. 2011). Buenos Aires. Recuperado de https://media. utp.edu.co/referencias-bibliograficas/uploads/referencias/ ponencia/263-la-escritura-en-la-investigacin-en-documentode-trabajo-no-19pdf-SVm6m-articulo.PDF

Carlino, P. (2012). Helping doctoral students of education to face writing and emotional challenges in identity transition. In M. Castelló \& C. Donahue (Eds.), University Writing: Selves and Texts in Academic Societies (pp. 217-234). London: Emerald Group Publishing Limited. https://doi.org/10.1163/9781780523873_013

Cerny, J. (1998). Historia de la Lingüistica. Cáceres, Esp.: Universidad de Extremadura. 
Cordeiro, Y., \& Lima, L. M. (2017). Publish and perish in the hands of predatory journals. Anais Academia Brasileira de Ciencias, 89(892), 787-788. https://doi.org/10.1590/0001-37652017892

Escandell, M. V. (1993). Introducción a la pragmática. Barcelona: Anthopos.

Fraser, J. F. (2017). Has "Publish or Perish" Become "Publish and Payment"? Navigating Neurosurgical Research in an Innovative Industry. World Neurosurgery, 104, 987-989. https://doi.org/10.1016/J. WNEU.2017.05.044

Galino, M. Á. (1960). Historia de la educación I: Edades antigua y media. Madrid: Gredos.

Garman, M. (1990). Psycholinguistics. Cambridge: Cambridge University Press. https://doi.org/10.1017/CBO9781139165914

Glänzel, W., \& Schoepflin, U. (1999). A bibliometric study of reference literature in the sciences and social sciences. Information Processing \& Management, 35(1), 31-44. https://doi.org/10.1016/ So306-4573(98)00028-4

Gooch, D., Vasalou, A., \& Benton, L. (2017). Impact in interdisciplinary and cross-sector research: Opportunities and challenges. Journal of the Association for Information Science and Technology, 68(2), 378-391. https://doi.org/10.1002/asi.23658

Goodman, S. N. (2018). A quality-control test for predatory journals. Nature, 553(7687): 155. https://doi: 10.1038/d41586-018-00403-Z

Hyland, K. (2011). Disciplines and discourses: social interactions in the construction of knowledge. In D. Starke-Meyerring, A. Paré, N. Artemeva, M. Horne, and L. Yousoubova (Eds.), Writing in the Knowledge Society. West Lafayette, IN: Parlor Press and The WAC Clearinghouse, 193-214.

Jacobs, J. A. (2013). In defense of disciplines : interdisciplinarity and specialization in the research university. Chicago: University of Chicago Press.

Lillis, T. (2003). Student Writing as “Academic Literacies": Drawing on Bakhtin to Move from Critique to Design. Language and Education, 17(3), 192-207. https://doi.org/10.1080/09500780308666848

Lotka, A. J. (1926). The frequency distribution of scientific productivity. Journal of Washington Academy Sciences, 16(12), 317-323. https:// doi.org/10.2307/24529203 
Mahecha, M. Á. (2008). La lingüística hoy: su (re)configuración a través de los diferentes objetos de estudio. Forma y Función, 21, 107-133.

Martínez-Miguélez, M. (2007). Conceptualización dela Transdisciplinariedad. Polis, Revista de La Universidad Bolivariana, 6(16), 1-17.

Mendoza, S., \& Paravic, T. (2006). Origen, clasificación y desafíos de las Revistas Científicas. Investigación y Postgrados, 21(1), 49-75.

Molina, B. G. (2017). Aportes de Saussure al desarrollo de la Lingüística como ciencia. Cuaderno de Pedagogía Universitaria, 14(28), 34-44.

Moreno Fernández, F. (1998). Principios de sociolingüística y sociología del lenguaje. Barcelona: Ariel.

Navarro, F., Ávila, N., Tapia-Ladino, M., Cristovão, V. L. L., Moritz, M. E. W., Narváez Cardona, E., \& Bazerman, C. (2016). Panorama histórico y contrastivo de los estudios sobre lectura y escritura en educación superior publicados en América Latina. Revista Signos, 49, 78-99. https://doi.org/10.4067/So718-09342016000400006

OECD. (2014). OECD work on Science, technology and industry (2014 No. 90061). París, Francia: OECD Publishing. Retrieved from http:// www.oecd.org/sti/oecd-science-technology-and-industry-outlook-19991428.htm

Pacheco, T. (2015). La tesis doctoral en ciencias sociales y su relación con el quehacer científico. Cinta Moebio, 52, 37-47.

Parodi, G. (2012). ¿Qué se lee en los estudios doctorales?: Estudio empírico basado en géneros a través del discurso académico de seis disciplinas. Revista de Lingüística Teórica y Aplicada, 50(2), 89119. https://doi.org/0033698X

Pfeiffer, R. (1981). Historia de la Filología Clásica. De los comienzos hasta el final de la época helenística. Madrid: Gredos.

Rawat, S., \& Meena, S. (2014). Publish or perish: Where are we heading? Journal of Research in Medical Sciences, 19(2), 87-89.

Riemer, N. (2010). Introducing semantics. Cambridge: Cambridge University Press.

Robins, R. (1997). Breve historia de la lingüistica. Madrid: Cátedra.

Salas, G., Ponce, F. P., Méndez-Bustos, P., Vega-Arce, M., Pérez, M. de los Á., López-López, W., \& Cárcamo-Vásquez, H. (2017). 25 Años de 
Psykhe: Un Análisis Bibliométrico. Psykhe, 26(1), 1-17. https:// doi.org/10.7764/psykhe.26.1.1205

Sánchez-Tarragó, N., Caballero-Rivero, A., Trzesniak, P., Deroy Domínguez, D., Nonato Macedo dos Santos, R., \& FernándezMolina, J.-C. (2016). Las revistas científicas en América Latina hacia el camino del acceso abierto: un diagnóstico de políticas y estrategias editoriales. TranInformacao, 28(2), 159-172. https:// doi.org/10.1590/2318-08892016000200003

Sarewitz, D. (2016). The pressure to publish pushes down quality. Nature, 533(7602): 147. https://doi.org/10.1038/533147a

Saussure, F. de. (2005). Cours de linguistique générale ( $3^{\circ}$ ed.). Genève: Arbre d'Or.

Schmitt, N. (2002). An Introduction to Applied Linguistics. London: Arnold.

Scopus. (2018). SJR. SCImago Journal Rank. Amsterdam: Elsevier. Retrieved from http://www.scimagojr.com/journalrank.php?category=12 o3\&area $=1200 \&$ type $=$ all

Takrouri, M. M. (2016). Publish or perish but do not publish and perish. Anesthesia: Essays and Researches, 10(2), 163. https://doi. org/10.4103/0259-1162.181225

Tasioulas, J., \& Vayena, E. (2016). The place of human rights and the common good in global health policy. Theoretical Medicine and Bioethics, 37(4), 365-382. https://doi.org/10.1007/s11017-016-9372-X

Valderrama, J. O. M. (2012). Aspectos éticos en las publicaciones de revistas científicas de corriente principal. Revista Chilena de Pediatria, 83(5), 417-419. https://doi.org/10.4067/ S0370-41062012000500001

Walsh, S. T. (2011). Publish or Perish: How Are Research and Reputation Related? Serials Review, 37(4), 244-257. https://doi.org/10.1080/ 00987913.2011.10765398

Xie, Z., Li, M., Li, J., Duan, X., \& Ouyang, Z. (2018). Feature analysis of multidisciplinary scientific collaboration patterns based on PNAS. EPJ Data Science, 7(5). https://doi.org/10.1140/epjds/ S13688-018-0134-Z

Zsiga, E. (2013). The Sounds of Language: An Introduction to Phonetics and Phonology. Malden, MA: Wiley-Blackwell. 
Zuin, A. A. S., Bianchetti, L., Zuin, A. A. S., \& Bianchetti, L. (2015). O produtivismo na era do "publique, apareça ou pereça": um equilíbrio difícil e necessário. Cadernos de Pesquisa, 45(158), 726-750. https://doi.org/10.1590/198053143294 